\title{
CIENCIAMATRIA
}

Revista Interdisciplinaria de Humanidades, Educación, Ciencia y Tecnología

Año VIII. Vol. VIII. No14. Enero - Junio. 2022

Hecho el depósito de ley: pp201602FA4721

ISSN-L: 2542-3029; ISSN: 2610-802X

Universidad Nacional Experimental Francisco de Miranda (UNEFM). Santa Ana de Coro. Venezuela

Cristhian Joel Guanín-Mackencie; Luís Orlando Albarracín-Zambrano; Dionisio Vitalio Ponce-Ruíz

DOI $10.35381 / \mathrm{cm} . v 8 \mathrm{i} 14.641$

\section{Sitio web adaptativo para la optimización en la gestión de ventas en el servicio funerario}

\section{Adaptive website for optimizing sales management in the funeral service industry}

Cristhian Joel Guanín-Mackencie

sq.cristhianjgm55@uniandes.edu.ec

Universidad Regional Autónoma de los Andes, Quevedo

Ecuador

https://orcid.org/0000-0002-4596-4482

Luís Orlando Albarracín-Zambrano

uq.luisalbarracin@uniandes.edu.ec

Universidad Regional Autónoma de los Andes, Quevedo

Ecuador

https://orcid.org/0000-0002-3164-5229

Dionisio Vitalio Ponce-Ruíz

manzanillo1962@gmail.com

Universidad Regional Autónoma de los Andes, Quevedo

Ecuador

https://orcid.org/0000-0002-5712-4376

Recibido: 15 de septiembre 2021

Revisado: 10 de noviembre 2021

Aprobado: 15 de diciembre 2021

Publicado: 01 de enero 2022 


\section{CIENCIAMATRIA \\ Revista Interdisciplinaria de Humanidades, Educación, Ciencia y Tecnología \\ Año VIII. Vol. VIII. №14. Enero - Junio. 2022 \\ Hecho el depósito de ley: pp201602FA4721 \\ ISSN-L: 2542-3029; ISSN: 2610-802X \\ Universidad Nacional Experimental Francisco de Miranda (UNEFM). Santa Ana de Coro. Venezuela}

Cristhian Joel Guanín-Mackencie; Luís Orlando Albarracín-Zambrano; Dionisio Vitalio Ponce-Ruíz

\section{Estimado Editor (a):}

La historia de la humanidad y su desarrollo se ha visto inmersa en epidemias que han formado parte habitual de los pueblos y civilizaciones dejando devastación y dolor. Esta pandemia del COVID-19 trastoca los cimientos de la sociedad del siglo XXI y pone en duda los sistemas sanitarios mundiales, la economía global plantea nuevos retos éticos y tecnológicos frente a las formas de cómo combatir este virus de manera efectiva (Mendieta, 2020).

En tal sentido, Ecuador es uno de los países de América Latina más golpeado por el COVID-19, actualmente el contagio comunitario aqueja a las 24 Provincias. Según el último reporte del Ministerio de Salud actualizado hasta la mañana del lunes 29 de noviembre del 2021. Los casos confirmados de Covid-19 en Ecuador ascienden a 526615 contagiados y 33219 muertes, cifras que fueron confirmadas por el Servicio Nacional de Gestión de Riesgos (Riesgos, 2020), Ecuador cuenta con cuatro tipos de cifras oficiales relacionadas con fallecimientos por covid-19 u otras causas:

En Quevedo, la crisis que afecta de igual manera que a nivel mundial hace crecer los requerimientos de servicios funerarios, y hace evidente la necesidad de implementar nuevas metodologías de gestión de ventas de los servicios que brindan las funerarias que desde hace décadas viene brindando y entre ellas la "Funeraria Guanín", y la pandemia del Covid 19 a virtualizado los servicios al cliente, esto ha hecho evidente el manejo de información a través de internet, y dispositivos móviles, las empresas deben ajustarse a esta tendencia de búsqueda de información online y la adquisición de un servicio o producto(caja fúnebre), el marketing digital es la herramienta necesaria para difundir los servicios que presta la empresa funeraria, al momento cuenta con difusión a través de medios escritos, y radiales únicamente, y la globalización de la información a través del internet, el uso de portales web que gestionen procesos, es una necesidad para llegar a una mayor cantidad de posibles clientes y satisfacer una necesidad. 


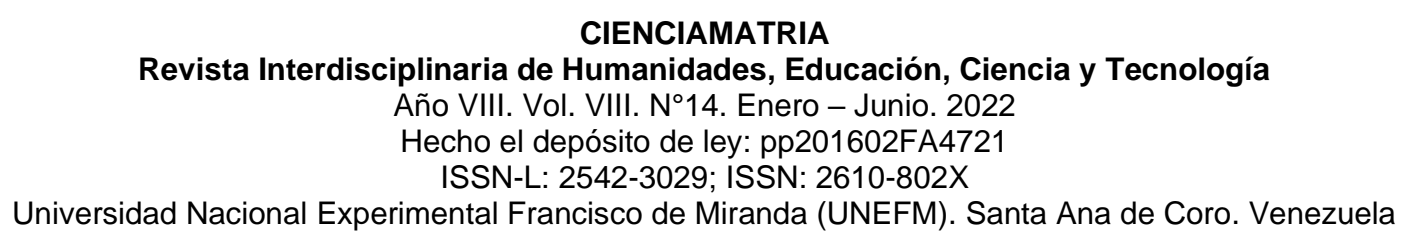

Cristhian Joel Guanín-Mackencie; Luís Orlando Albarracín-Zambrano; Dionisio Vitalio Ponce-Ruíz

La empresa funeraria Guanín pese a mantenerse por años en el mercado no cuenta con sistema automatizado que permita la generación de facturas, lo cual hace que se traspapelen o pierdan ciertas facturas, o al momento de elaborarlas se cometa errores humanos de escritura lo cual hace que se deban anular. Se empieza a desarrollar teorías, modelos de cómo aplicar la gestión comercial (ventas) por internet, que se han convertido en medio de difusión general entre la población, la gestión de ventas se encargan de los intercambios de información desde una óptica en la cual las promociones y servicios pueden darse entre cualquier tipo de público dicho del sector público o privado, sin sectorizar su estrato social.

Para Bagaña Samaniego (2019), finalmente, los servicios exequiales en Ecuador se han posicionado en el mercado, la cual se ha ido innovando de acuerdo con la tendencia, desde entonces nació varios negocios funerarios con una proyección empresarial amplia. Por ello, la creación de diversas empresas dedicadas a la prestación de servicios fúnebres. El sector funerario se entiende el lugar o sitio donde los seres queridos dejan a una persona que ha fallecido por alguna enfermedad u otra razón

Por otro lado, Cañón Rubiano (2020) plantea que aceptar la muerte no resulta fácil, pero se podría decir que la actitud como se toma es distinta y determinada por la cultura donde se habita. La búsqueda de sistemas simbólicos complejos que busquen el control del duelo, o el rito funerario, los rituales funerarios constituyen actividades humanas que se ponen en práctica a fin de expresar, a través de ellos, la complejidad de símbolos existentes en torno a la concepción sobre la vida y la muerte. Involucran, por ende, tanto al difunto como a sus deudos.

Sin embargo, las ventas online están creciendo, aún existen muchas personas que no se sienten seguras de realizarlas por este medio, por lo que prefieren ir a la tienda o en su defecto darle un "rostro" a quien están comprando; es decir hacer su primera compra en la tienda y después si por internet, que es lo que sucede en la mayoría de los casos. (Rodríguez et al., 2017) 


\section{CIENCIAMATRIA}

Revista Interdisciplinaria de Humanidades, Educación, Ciencia y Tecnología

Año VIII. Vol. VIII. №14. Enero - Junio. 2022

Hecho el depósito de ley: pp201602FA4721

ISSN-L: 2542-3029; ISSN: 2610-802X

Universidad Nacional Experimental Francisco de Miranda (UNEFM). Santa Ana de Coro. Venezuela

Cristhian Joel Guanín-Mackencie; Luís Orlando Albarracín-Zambrano; Dionisio Vitalio Ponce-Ruíz

La situación de hoy en día por la pérdida de familiares a consecuencia de la pandemia del Covid 19 es un afecto es alarmante, y la empresa la evidencia pues la intensa competencia con otras empresas de fuera o dentro del catón que aplican la mercadotecnia abaratando costos, pero sin brindar servicios profesionales de tratamiento enmarcados en las exigencias de los órganos reguladores sobre el tratamiento de los cadáveres de una manera sanitaria, otorga cierta desventaja, que acentúa el decrecimiento del servicio, por ello es necesario reforzar aspectos potenciales que se difunde mediante la aplicación de estrategias online con un sitio web de gestión de ventas, sugeridas por el mercado el cual se capta con un mayor número de clientes asegurando incrementar la cartera de clientes y satisfacer la demanda del servicio funerario y ampliando el mercado para la funeraria "Guanin".

Una solución informática mediante la programación web aplicando una metodología de desarrollo rápido, con una interfaz de fácil manejo para los usuarios, mediante el cual aceden a la información de los productos y servicios que brinda la empresa funeraria "Guanin", permitiéndoles registrarse datos que son importantes para los propietarios y administrador del sistema para las estadísticas de clientes y visitantes, los clientes pueden acceder a realizar una adquisición o contratación de servicios mediante un carrito de compras, lo cual es una gran ventaja hoy en día con los nuevos índices de contagios del Covid19 y en este inicio del año 2022 se ha visto el nuevo oleaje de casos positivos, colocando a Quevedo el 22 de enero del 2022 en semáforo en rojo con nuevas pérdidas humanas, este sistemas presenta su novedad por ser la primera plataforma virtual en el cantón Quevedo, que permite el acceso a clientes de funerarias a contratar sin arriesgar contactos personales para ello.

Por lo tanto, la gestión de ventas se analiza con el objeto de comprender el mercado informativo social, establecido en el área administrativa de marketing y ventas como organización, permitiendo que los usuarios accedan a la interacción que existe con el cliente, su propósito es dar a brindar un mejor servicio de una forma innovadora. 


\begin{abstract}
CIENCIAMATRIA
Revista Interdisciplinaria de Humanidades, Educación, Ciencia y Tecnología

Año VIII. Vol. VIII. N¹4. Enero - Junio. 2022

Hecho el depósito de ley: pp201602FA4721

ISSN-L: 2542-3029; ISSN: 2610-802X

Universidad Nacional Experimental Francisco de Miranda (UNEFM). Santa Ana de Coro. Venezuela

Cristhian Joel Guanín-Mackencie; Luís Orlando Albarracín-Zambrano; Dionisio Vitalio Ponce-Ruíz
\end{abstract}

Aplicando las etapas de gestión de ventas como, por ejemplo: planeación, clasificación, combinación, entre otras etapas. La situación en tiempos de Covid19 que afectan a las microempresas permite evidenciar la pertinencia del desarrollo de proyectos, permitiendo establecer una estrategia tecnológica para la solución de los problemas.

\title{
FINANCIAMIENTO
}

No monetario.

\section{AGRADECIMIENTO}

A la Universidad Regional Autónoma de los Andes, Quevedo, por motivar el desarrollo de la Investigación.

\section{REFERENCIAS CONSULTADAS}

Bajaña Mendieta, I. de la L. (2020). Incidencias del Covid-19 en Ecuador. [Incidences of Covid-19 in Ecuador]. Question/Cuestión, 1 (mayo), e321. https://doi.org/10.24215/16696581e321

Cañon Rubiano, C. A. (2020). Arquitectura funeraria: el encuentro de atmósferas de contraste en el rito funerario. [Funerary architecture: the encounter of contrasting atmospheres in the funeral rite] Trabajo de grado. Universidad de Bogotá Jorge Tadeo Lozano. Bogota. Colombia. Recuperado de: https://n9.cl/7zzt1

Riesgos, S. N. (21 de agosto de 2020). Infografia-Nacionalcovi-19-Coe-Nacional. [Infographic-Nationalcovi-19-Coe-National] Recopilado de: https://n9.cl/aa6417

Rodríguez, R., Marko, I., Vera,P., Vallés, G., Acevedo Zain, G.(2017).Evaluación de la Usabilidad en Sitios Web Móviles Adaptativos. [Usability Assessment on Adaptive Mobile Websites]. Recuperado de: https://n9.cl/b9qka 


\section{CIENCIAMATRIA}

Revista Interdisciplinaria de Humanidades, Educación, Ciencia y Tecnología

Año VIII. Vol. VIII. №14. Enero - Junio. 2022

Hecho el depósito de ley: pp201602FA4721

ISSN-L: 2542-3029; ISSN: 2610-802X

Universidad Nacional Experimental Francisco de Miranda (UNEFM). Santa Ana de Coro. Venezuela

Cristhian Joel Guanín-Mackencie; Luís Orlando Albarracín-Zambrano; Dionisio Vitalio Ponce-Ruíz

Samaniego, L. (2019). Estudio de factibilidad para la creación de una funeraria, ubicada en el sur del distrito metropolitano de quito, año 2019. [Feasibility study for the creation of a funeral home, located in the south of the metropolitan district of Quito, year 2019]. (Bachelor's thesis).Tecnológico Superior Cordillera. Quito. Recuperado de: https://n9.cl/yrojp

Vaczi, M. (2019). Muerte en la Catedral: Cenizas y ritos funerarios en los campos de fútbol. [Death in the Cathedral: Ashes and funeral rites on the football fields] Disparidades. Revista de Antropología, 74(2), e020. https://doi.org/10.3989/dra.2019.02.020

(C2022 por los autores. Este artículo es de acceso abierto y distribuido según los términos y condiciones de la licencia Creative Commons Atribución-NoComercial-Compartirlgual 4.0 Internacional (CC BY-NC-SA 4.0) (https://creativecommons.org/licenses/by-nc-sa/4.0/). 